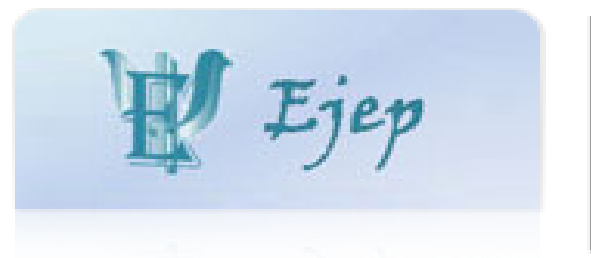

\author{
European Journal of Education and \\ Psychology \\ ISSN: 1888-8992 \\ ejep@ejep.es \\ Editorial CENFINT \\ España
}

Torregrosa, María S.; Inglés, Cándido J.; García-Fernández, José M.; Ruiz-Esteban, Cecilia; López-

García, Karla S.; Zhou, Xinyue

Diferencias en conducta agresiva entre adolescentes españoles, chinos y mexicanos

European Journal of Education and Psychology, vol. 3, núm. 2, diciembre, 2010, pp. 167-176

Editorial CENFINT

Almería, España

Disponible en: http://www.redalyc.org/articulo.oa?id=129315468001

- Cómo citar el artículo

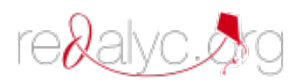

- Número completo

- Más información del artículo

Página de la revista en redalyc.org

Sistema de Información Científica

Red de Revistas Científicas de América Latina, el Caribe, España y Portugal Proyecto académico sin fines de lucro, desarrollado bajo la iniciativa de acceso abierto 


\title{
Diferencias en conducta agresiva entre adolescentes españoles, chinos y mexicanos
}

\author{
María S. Torregrosa ${ }^{1}$, Cándido J. Inglés ${ }^{1}$, José M. García-Fernández ${ }^{2}$, Cecilia \\ Ruiz-Esteban ${ }^{3}$, Karla S. López-García y Xinyue Zhou ${ }^{5}$ \\ ${ }^{1}$ Universidad Miguel Hernández de Elche, ${ }^{2}$ Universidad de Alicante, \\ ${ }^{3}$ Universidad de Murcia, (España), ${ }^{4}$ Universidad Autónoma de Nuevo León (México), \\ ${ }^{5}$ Sun Yat-Sen University (China)
}

\begin{abstract}
La evidencia empírica ha revelado diferencias culturales en la expresión de la agresividad. El objetivo de este estudio fue analizar las diferencias de agresividad entre estudiantes españoles, mexicanos y chinos de Educación Secundaria. La escala de Conducta Antisocial del Teenage Inventory of Social Skills (TISS) fue administrada a 420 españoles, 532 mexicanos y 431 chinos, con edades comprendidas entre los 12 y los 15 años. Los análisis de varianza mostraron que los estudiantes chinos presentaron niveles significativamente más altos de agresividad que los estudiantes españoles y mexicanos, no encontrándose diferencias entre los dos últimos grupos. Este patrón de resultados fue similar por género y edad. Así, todas las submuestras de estudiantes chinos (chicos, chicas, 12-13 años y 14-15 años) informaron niveles significativamente más altos de agresividad que sus iguales españoles y mexicanos. Sin embargo, las diferencias encontradas fueron de magnitud pequeña y moderada. Los resultados son discutidos de acuerdo con las dimensiones culturales de individualismo vs. colectivismo y distancia de poder.
\end{abstract}

Palabras clave: Diferencias transculturales, agresividad, adolescencia, sexo, edad, educación secundaria.

Empirical evidence has revealed cultural differences in the expression of aggressiveness. The purpose of this study was to analyse aggressive behaviour differences among Spanish, Mexican and Chinese students of secondary education. The Antisocial Behaviour scale of the Teenage Inventory of Social Skills (TISS) was administered to 420 Spanish, 532 Mexican, and 431 Chinese, ranging from 12 to 15 years. Variance analyses showed that Chinese students presented significant higher levels of aggressiveness than Spanish and Mexican students. No differences were found among the last two groups. This pattern of results was similar by gender and age groups. Thus, all subsamples of Chinese students (boys, girls, 12-13 year olds, and 14-15 year olds) reported significant higher levels of aggressiveness than their Spanish and Mexican peers. However, differences found were of small and moderate magnitude. Results are discussed according to cultural dimensions of individualism vs. collectivism and power distance.

Key words: Cross-cultural differences, aggressiveness, adolescence, gender, age, secondary education.

Correspondencia: Cándido J. Inglés. Área de Psicología Evolutiva y de la Educación. Dpto. de Psicología. Universidad Miguel Hernández. Avda. de la Universidad, s/n. 03202 Elche, Alicante (España). E-mail: cjingles@umh.es 
La agresividad se define como toda aquella conducta dirigida hacia otra persona con la intención de causar daño (Anderson y Bushman, 2002). Sin embargo, la forma de expresar la agresividad, así como la tolerancia hacia las conductas agresivas, varía en las diferentes sociedades (Fry, 2001). Entre los modelos existentes para la explicación de la agresividad, el modelo de agresión general (General Aggression Model, GAM), propuesto por Anderson y Bushman (2002), señala la influencia de los valores y creencias del individuo en su actuación agresiva. Dado que estos valores y creencias están socialmente determinados (Páez, Fernández, Basabe y Grad, 2001), parece indiscutible el papel determinante que juega la cultura en las manifestaciones agresivas.

Esta relación entre la cultura propia de una sociedad y la agresión se ha visto reflejada en diferentes estudios. Concretamente, Crijnen, Achenbach y Verhulst (1999) señalaron que las diferencias culturales permitían explicar el 5\% de la varianza de las conductas agresivas de niños y adolescentes informadas por los padres. Otros estudios han puesto de manifiesto que, si bien existe un grado de aceptación similar en cuanto a la agresividad interpersonal entre estudiantes de diferentes culturas, aparecen diferencias respecto a las actividades agresivas aceptadas y las circunstancias en las que se justifican (Ramírez, 1993). Este autor señala, en contraposición con los resultados obtenidos para estudiantes polacos y finlandeses, que los universitarios españoles presentaban una mayor aceptación de las conductas agresivas con elevada carga emocional (ej. gritar o actuar de forma irónica) y en las que estaban implicados sus propios intereses. Estudios posteriores (Fujihara, Kohyama, Andreu y Ramírez, 1999) muestran también diferencias en lo referente a las actividades agresivas aceptadas entre universitarios españoles, estadounidenses y japoneses, siendo estos últimos los que presentaban una mayor justificación de la agresión verbal directa (entendida como gritar o protestar furiosamente) y una menor justificación de la agresión verbal indirecta (entendida como actuar de forma irónica o interferir o impedir la actuación de otra persona).

También existen otros factores personales, como el sexo y la edad, que han demostrado estar implicados en las diferencias encontradas en la interpretación y actuación agresiva de los individuos de distintas sociedades, encontrándose resultados dispares. Así, mientras en la comparación de la sociedad japonesa y la española se encuentran diferencias en agresividad física entre las mujeres a favor de las japonesas, sin encontrarse diferencias entre los varones (Ramírez, Andreu y Fujihara, 2001); al comparar a estudiantes italianos, húngaros y checos se encuentran diferencias entre los varones a favor de los italianos, pero no aparecen diferencias entre las mujeres (Pastorelli, Barbaranelli, Cermark, Rozsa y Caparara, 1997). Estudios recientes en los que se comparan las sociedades china, polaca y estadounidense muestran diferencias en agresividad directa e indirecta tanto en hombres como en mujeres, siendo los chinos quienes informan niveles más bajos en ambos tipos de agresividad (Forbes, Zhang, 
Doroszewicz y Haas, 2009). En relación a la edad, Österman et al. (1998), en un estudio con adolescentes de diversas culturas, destacaron la existencia de diferencias en el tipo de agresión utilizada por chicos y chicas de diferentes edades. Mientras que en la infancia y preadolescencia ( 8 y 11 años) las chicas informaban más conductas de agresividad indirecta, los chicos de la misma edad presentan mayor agresividad física y verbal; al aumentar la edad (15 años) tanto los chicos como las chicas utilizaban en mayor medida la agresividad indirecta que cualquier otro tipo de agresión.

En estos momentos, y pese a la existencia de diferentes estudios que señalan la importancia de tener en cuenta las conductas agresivas durante la adolescencia (Estévez, Murgui y Musitu, 2008; Inglés et al., 2008; Toldos, 2005), las investigaciones en las que se aborda la agresividad desde una perspectiva intercultural con población adolescente no clínica son escasas (Crijnen et al., 1999; Österman et al., 1998; Pastorelli et al., 1997). La presente investigación tiene como fin aportar datos empíricos al estudio de la agresividad en población adolescente, siendo el objetivo principal el análisis de las diferencias en conductas agresivas de estudiantes españoles, mexicanos y chinos. En el estudio meta-analítico de Bergeron y Schneider (2005) se evaluaron los niveles de agresividad en los individuos de estas tres sociedades en relación con la sociedad norteamericana, destacando que los mexicanos presentaban niveles más altos de agresividad que los norteamericanos, mientras que españoles y chinos informaban niveles menores de agresividad. Tal y como apuntan estos autores, y atendiendo a la clasificación de las culturas como individualistas-colectivistas, es más probable que los individuos pertenecientes a culturas individualistas presenten más conductas agresivas, en tanto en cuanto estas acciones les permitan alcanzar sus propias metas. Por el contrario, aquellos individuos pertenecientes a culturas colectivistas dan mayor importancia a la pertenencia al grupo y al bien común, con lo que probablemente presentarán menos actos agresivos. Atendiendo a esta diferenciación y congruentemente con los datos de investigaciones anteriores, se espera que los adolescentes chinos presenten niveles de agresividad significativamente inferiores a los de los estudiantes españoles y mexicanos, dado que se considera que China es un claro ejemplo de sociedad colectivista (Oyserman, Coon y Kemmelmeier, 2002), mientras que España y México son reflejo de sociedades mixtas, presentando rasgos colectivistas e individualistas (Correa, Contreras, Ramírez y López, 2002; Gouveia, 1998). Asimismo, ya que el sexo y la edad han demostrado ser variables influyentes en la investigación transcultural sobre agresividad, se analizan las diferencias entre los adolescentes chinos, españoles y mexicanos atendiendo al sexo y la edad. En este sentido, se espera que tanto en los análisis referidos al sexo como en los referidos a la edad se mantengan los mismos patrones que los esperados para la población general. 


\section{METODO}

\section{Participantes}

Para la realización de este trabajo se realizó un muestreo no probabilístico por conveniencia. La muestra reclutada fue de 1.383 adolescentes de 12 a 15 años de España, México y China.

La muestra española estaba formada por 420 estudiantes (varones $N=215$, mujeres $N=205)$, con una media de edad de $14.32(D T=1.23)$. La muestra mexicana la formaron 532 estudiantes (varones $N=229$; mujeres $N=303$ ), con una media de edad de $14.13(D T=0.93)$. La muestra china se compuso de 431 estudiantes (varones $N=230$, mujeres $N=201)$, con una media de edad de $14.35(D T=1.20)$.

La clasificación de los participantes en función del género y la edad para cada país (España, México y China) se muestra en la tabla 1.

\begin{tabular}{ccccc}
\multicolumn{2}{c}{ Tabla 1. Número de sujetos de la muestra clasificados por país, sexo y edad } \\
\cline { 3 - 5 } & & $12-13$ & $14-15$ & Total \\
España & Varones & 73 & 142 & 215 \\
& Mujeres & 77 & 128 & 205 \\
\hline \multirow{2}{*}{ México } & Varones & 65 & 164 & 229 \\
& Mujeres & 82 & 221 & 303 \\
\hline \multirow{2}{*}{ China } & Varones & 71 & 159 & 230 \\
& Mujeres & 72 & 129 & 201 \\
\hline \multirow{2}{*}{ Total } & & 440 & 943 & 1.383 \\
\hline
\end{tabular}

Las pruebas $\chi^{2}$ de homogeneidad de la distribución de frecuencias revelaron la ausencia de diferencias estadísticamente significativas entre los cuatro grupos Sexo $\mathrm{x}$ Edad en España $\left(\chi^{2}=0.595, p=0.44\right)$, México $\left(\chi^{2}=0.114, p=0.74\right)$ y China $\left(\chi^{2}=1.186\right.$, $p=0.28)$. Tampoco se encontraron diferencias estadísticamente significativas en cuanto a la homogeneidad de la distribución entre los grupos Sexo x Edad x País $\left(\chi^{2}=0.813\right.$, $p=0.85)$.

\section{Instrumentos}

El instrumento de evaluación empleado fue el Inventario de Habilidades Sociales para Adolescentes (Teenage Inventory of Social Skills, TISS; Inderbitzen y Foster, 1992).

Esta prueba evalúa la competencia social de los adolescentes en las relaciones con sus iguales. Consta de 40 ítems valorados en una escala Likert de seis puntos $(1=$ no me describe nada; $6=$ me describe totalmente). Está compuesta por dos escalas: Conducta Prosocial y Conducta Antisocial, cuyas puntuaciones se obtienen sumando los valores asignados por los sujetos a los ítems que componen cada dimensión. Puntuaciones altas indican una elevada conducta prosocial o antisocial. 
Inglés, Hidalgo, Méndez e Inderbitzen (2003) analizaron las propiedades psicométricas de la prueba en población adolescente hispanoparlante, obteniendo resultados satisfactorios y semejantes a los informados por Inderbitzen y Foster (1992) en población adolescente norteamericana.

Para este estudio se utilizó la puntuación obtenida en la escala de Conducta Antisocial del TISS, siendo los coeficientes de consistencia interna (alpha de Cronbach) de $0.83,0.84$ y 0.87 para población española, mexicana y china, respectivamente.

\section{Procedimiento}

La adaptación china del TISS se llevó a cabo siguiendo un proceso de retrotraducción (para más información ver Hambleton y Kanjee, 1995), no siendo necesaria la eliminación de ningún ítem o un cambio sustancial en los mismos durante el proceso de traducción.

Una vez traducida la escala para población china, se llevó a cabo una entrevista con los directores y orientadores de los centros participantes con el fin de exponer los objetivos del estudio, solicitar su permiso y promover su colaboración. Posteriormente, se explicó el estudio a los padres, solicitando su aprobación por escrito para la participación de sus hijos en la investigación.

Antes de la cumplimentación del cuestionario se pidió a los estudiantes que completaran los datos de identificación (sexo, edad, curso, grupo, centro y país de origen). Una vez cumplimentados estos datos, se leyeron en voz alta las instrucciones, recalcando la importancia de no dejar ninguna pregunta sin contestar. Los cuestionarios fueron contestados de forma colectiva, voluntaria y anónima en el aula. Los investigadores estuvieron presentes durante la administración de las pruebas para aclarar posibles dudas y verificar la correcta cumplimentación del cuestionario.

\section{Análisis de datos}

Se realizaron análisis de varianza (ANOVA) para analizar la existencia de diferencias culturales en la escala Conducta Antisocial entre los estudiantes españoles, mexicanos y chinos, así como para evaluar estas diferencias en función del género y la edad. Para identificar entre qué grupos se hallaban las diferencias se llevaron a cabo comparaciones post hoc (Prueba de Scheffé). Asimismo, se calculó el tamaño del efecto o diferencia media tipificada (índice $d$ ), con el fin de determinar la magnitud de las diferencias halladas por la prueba $F$. La interpretación del tamaño del efecto es la siguiente: (a) valores menores o iguales a 0.20 indican un tamaño del efecto insignificante; (b) valores entre 0.20 y 0.49 señalan un tamaño del efecto pequeño; (c) valores entre 0.50 y 0.79 muestran un tamaño del efecto medio o moderado; y (d) valores iguales o mayores a 0.80 indican un tamaño del efecto grande (Cohen, 1988). 


\section{RESULTADOS}

\section{Diferencias culturales en agresividad}

Los datos señalaron diferencias estadísticamente significativas entre los estudiantes españoles, mexicanos y chinos en las puntuaciones de la escala Conducta Antisocial, tal y como se muestra en la tabla 2.

Las pruebas post hoc permitieron comprobar que, mientras no aparecían diferencias estadísticamente significativas entre estudiantes españoles y mexicanos, los estudiantes chinos presentaban puntuaciones significativamente más elevadas en agresividad que los estudiantes españoles $(d=-0.47)$ y mexicanos $(d=-0.46)$, siendo la magnitud de estas diferencias pequeña.

Diferencias culturales en función del sexo

$\mathrm{Al}$ evaluar la conducta agresiva en las diferentes culturas teniendo en cuenta el sexo, los resultados también informaron de diferencias estadísticamente significativas tanto entre los varones como entre las mujeres de los tres países (Tabla 2).

Los análisis posteriores permitieron comprobar que no existían diferencias estadísticamente significativas entre varones españoles y mexicanos. Sin embargo, los varones chinos presentaban puntaciones significativamente más altas que los españoles $(d=-0.25)$ y mexicanos $(d=-0.35)$, siendo pequeño el tamaño de estas diferencias.

En el caso de las mujeres se observó una tendencia similar a la encontrada para los varones. No se encontraron diferencias estadísticamente significativas entre mujeres españolas y mexicanas, mientras que las mujeres chinas presentaron puntuaciones significativamente más altas en agresividad que las estudiantes españolas $(d=-0.71)$ y mexicanas $(d=-0.54)$, siendo el tamaño de estas diferencias de magnitud moderada.

Diferencias culturales en función de la edad

$\mathrm{Al}$ analizar los resultados en función de la edad se comprobó que en este caso también aparecían diferencias estadísticamente significativas entre los estudiantes de 12-13 años (adolescencia temprana) y entre los estudiantes de 14-15 años (adolescencia media) de España, México y China (Tabla 2).

Los análisis post hoc permitieron comprobar que no existían diferencias estadísticamente significativas entre los estudiantes españoles y mexicanos de 12-13 años. Por otra parte, los estudiantes chinos de esta edad informaron más conductas agresivas que los estudiantes españoles $(d=-0.65)$ y mexicanos $(d=-0.53)$, siendo la magnitud de estas diferencias moderada.

Finalmente, en los estudiantes de 14-15 años se halló una tendencia similar a la encontrada con los adolescentes más jóvenes. En este caso tampoco se observaron diferencias estadísticamente significativas entre estudiantes españoles y mexicanos, aunque las comparaciones con los estudiantes chinos demostraron que estos últimos 
informaban conductas significativamente más agresivas que los adolescentes españoles $(d=-0.38)$ y mexicanos $(d=-0.43)$ de la misma edad. Los tamaños del efecto para estas diferencias fueron de pequeña magnitud.

\begin{tabular}{|c|c|c|c|c|c|c|c|c|c|c|c|c|}
\hline & & \multirow{2}{*}{$\begin{array}{c}\text { España } \\
M(D T) \\
\end{array}$} & \multirow{2}{*}{$\begin{array}{l}\text { México } \\
M(D T) \\
\end{array}$} & \multirow{2}{*}{$\begin{array}{c}\text { China } \\
M(D T) \\
\end{array}$} & \multicolumn{2}{|c|}{$\begin{array}{c}\text { Significación } \\
\text { estadística }\end{array}$} & \multicolumn{2}{|c|}{$\begin{array}{l}\text { España/ } \\
\text { México }\end{array}$} & \multicolumn{2}{|c|}{ España/China } & \multicolumn{2}{|c|}{ México/China } \\
\hline & & & & & $F_{(2,1380)}$ & $p$ & $p$ & $d$ & $p$ & $d$ & $p$ & $d$ \\
\hline $\begin{array}{l}\text { Conducta } \\
\text { Antisocial } \\
\text { (TISS) }\end{array}$ & $\begin{array}{l}\text { Población } \\
\text { total }\end{array}$ & $\begin{array}{c}49.77 \\
(13.81)\end{array}$ & $\begin{array}{c}49.27 \\
(16.40)\end{array}$ & $\begin{array}{c}56.73 \\
(15.70)\end{array}$ & 32.801 & 0.000 & n.s. & - & 0.000 & -0.47 & 0.000 & 0.46 \\
\hline \multirow{2}{*}{$\begin{array}{l}\text { Conducta } \\
\text { Antisocial } \\
\text { (TISS) }\end{array}$} & Varones & $\begin{array}{c}53.76 \\
(12.82)\end{array}$ & $\begin{array}{c}51.70 \\
(16.46)\end{array}$ & $\begin{array}{c}57.32 \\
(15.50)\end{array}$ & 8.162 & 0.000 & n.s. & - & 0.046 & -0.25 & 0.000 & -0.35 \\
\hline & Mujeres & $\begin{array}{c}45.58 \\
(13.59) \\
\end{array}$ & $\begin{array}{c}47.42 \\
(16.14) \\
\end{array}$ & $\begin{array}{c}56.06 \\
(15.94) \\
\end{array}$ & 27.658 & 0.000 & n.s. & - & 0.000 & -0.71 & 0.000 & -0.54 \\
\hline \multirow{2}{*}{$\begin{array}{l}\text { Conducta } \\
\text { Antisocial } \\
\text { (TISS) }\end{array}$} & $\begin{array}{l}12-13 \\
\text { años }\end{array}$ & $\begin{array}{c}48.83 \\
(13.83)\end{array}$ & $\begin{array}{c}49.59 \\
(17.70)\end{array}$ & $\begin{array}{c}58.47 \\
(15.92)\end{array}$ & 16.496 & 0.000 & n.s. & - & 0.000 & -0.65 & 0.000 & -0.53 \\
\hline & $\begin{array}{l}14-15 \\
\text { años }\end{array}$ & $\begin{array}{c}50.29 \\
(13.79)\end{array}$ & $\begin{array}{c}49.14 \\
(15.90)\end{array}$ & $\begin{array}{c}55.87 \\
(15.55)\end{array}$ & 17.352 & 0.000 & n.s. & - & 0.000 & -0.38 & 0.000 & -0.43 \\
\hline
\end{tabular}

Nota. n.s. = no significativo

\section{DISCUSION}

El objetivo principal de la presente investigación fue el análisis de las diferencias en conductas agresivas entre adolescentes españoles, mexicanos y chinos. Los resultados han señalado que, si bien existen diferencias entre los estudiantes de las tres culturas, estas diferencias no se presentan en la dirección esperada. Se hipotetizó inicialmente que los adolescentes chinos informarían menos conductas agresivas que los adolescentes españoles y mexicanos, tanto en la población general como al llevar a cabo los análisis en función del género y la edad. Sin embargo, los resultados apuntan en la dirección contraria, siendo los adolescentes chinos los más agresivos. Estos resultados se muestran incongruentes con los presentados en investigaciones previas (Bergeron y Schneider, 2005; Forbes et al., 2009), en las que la población china presentaba niveles inferiores de agresividad que otras culturas, relacionándose estos bajos niveles de agresividad con su pertenencia a una sociedad altamente colectivista, en la que se da gran importancia a la pertenencia al grupo y a la búsqueda del bien común. Sin embargo, en otras sociedades altamente colectivistas, como Japón (Hofstede, 1984), se han obtenido resultados congruentes, en parte, con el presente estudio en cuanto a los comportamientos agresivos. Concretamente, Ramírez et al. (2001) señalan que los universitarios japoneses informan mayores niveles de agresividad física, mientras que los españoles informan mayores niveles de agresividad verbal.

Entre las posibles explicaciones de los resultados obtenidos se considera que los mayores niveles de agresividad autoinformada por los estudiantes chinos podrían estar relacionados con el instrumento utilizado, ya que, pese a que la escala mostró una 
adecuada consistencia interna para esta población, la prueba fue concebida para evaluar el comportamiento interpersonal de adolescentes norteamericanos (Inderbitzen y Foster, 1992), y por tanto, puede que en el cuestionario se reflejen aspectos que no necesariamente concuerden con las conductas de los adolescentes chinos. Los resultados obtenidos también pueden indicar la importancia de tener en consideración otras posibles explicaciones de las diferencias interculturales que van más allá de la dimensión individualista-colectivista. Hofstede (1984) relaciona la dimensión individualismocolectivismo con la dimensión de distancia al poder. Según el estudio meta-analítico realizado por Bergeron y Schneider (2005), la dimensión de distancia al poder presenta una mayor contribución a la explicación de las diferencias interculturales debidas a las diferencias en los valores en las distintas culturas que el individualismo-colectivismo. Así, se constata que una menor distancia al poder, entendida como la aceptación de los individuos como iguales y la participación voluntaria en la sociedad, contribuye a que los niveles de agresividad sean menores. Atendiendo a esta dimensión, sería posible que las diferencias encontradas pudieran ser explicadas en base a esta mayor distancia al poder de la sociedad china en contraposición con la española. Son necesarias, sin embargo, nuevas investigaciones en esta línea, que estudien con mayor profundidad la relación entre las sociedades española, mexicana y china, y aporten luz sobre las posibles causas de estas diferencias. Asimismo, sería necesario que futuras investigaciones examinen cómo se producen estas influencias culturales en agresividad. En este sentido, estudios recientes han destacado que las conductas agresivas de los adolescentes se relacionan con su autoconcepto (De la Torre, García, Carpio y Casanova, 2008). Así, puesto que la formación del autoconcepto se basa tanto en factores cognitivos como en la interacción social (Shavelson, Hubner y Stanton, 1976), es posible que la influencia de la cultura sobre las conductas agresivas esté mediada por su acción sobre otras variables (por ej. el autoconcepto).

Este estudio presenta varias limitaciones que deberían tenerse en cuenta en futuras investigaciones. Por un lado, el instrumento utilizado no permite analizar diferencias entre distintas manifestaciones de agresividad. Dado que se ha comprobado la existencia de diferencias culturales en función del tipo de conducta agresiva (Bergeron y Schneider, 2005; Crijnen et al., 1999; Forbes et al., 2009; Pastorelli et al., 1997; Ramírez et al., 2001), sería conveniente que en futuras investigaciones se utilicen instrumentos que permitan evaluar estas diferencias. Por otro lado, el uso de autoinformes para la evaluación de la conducta agresiva puede resultar problemático, puesto que este tipo de conductas, al no ser socialmente aceptadas, tienden a encubrirse. Con el fin de solventar este problema sería recomendable la utilización de diferentes formas de evaluación, incluyéndose tanto la observación como los informes de otros significativos, lográndose así un análisis mucho más exhaustivo de la conducta problemática. 
Pese a estas limitaciones y a que los resultados no hayan permitido apoyar las hipótesis de partida, se considera que la presente investigación supone una contribución importante en el estudio de las diferencias culturales en agresividad, aportando datos novedosos y enmarcando el estudio de la interculturalidad dentro de una perspectiva teórica que explica los fenómenos agresivos (modelo de agresión general), siendo además uno de los pocos estudios que tienen en cuenta estas diferencias en población adolescente.

\section{Agradecimientos}

Este trabajo ha sido realizado a través del Proyecto SEJ 2004-07311/EDUC perteneciente al Plan Nacional de Investigación Científica, Desarrollo e Innovación Tecnológica del M.E.C. concedido al segundo autor.

\section{REFERENCIAS}

Anderson, C.A. y Bushman, B.J. (2002). Human aggression. Annual Review of Psychology, 53, 27-51.

Bergeron, N. y Schneider, B.H. (2005). Explaining cross-national differences in peer-directed aggression: A quatitative synthesis. Aggressive Behavior, 31(2), 116-137.

Cohen, J. (1988). Statistical power analysis for the behavioral sciences (2nd Ed.). Hillsdale, NJ, USA: Erlbaum.

Correa, F., Contreras, C., Ramírez, A. y López, E. (2002). Dimensiones del individualismocolectivismo en México: Un estudio exploratorio. La Psicología Social en México, 9, 553-559.

Crijnen, A.A.M., Achenbach, T.M. y Verhulst, F.C. (1999). Problems reported by parents of children in multiple cultures: The Child Behavior Checklist syndrome constructs. The American Journal of Psychiatry, 156(4), 569-574.

De la Torre, M.J., García, M. C., Carpio, M.V. y Casanova, P.F. (2008). Relaciones entre violencia escolar y autoconcepto multidimensional en adolescentes de Educación Secundaria Obligatoria. European Journal of Education and Psychology, 1(2), 57-70.

Estévez, E., Murgui, S. y Musitu, G. (2008). Psychosocial adjustment in aggressors, pure victims and aggressive victims at school. European Journal of Education and Psychology, 1(2), 29-39.

Forbes, G., Zhang, X., Doroszewicz, K. y Haas, K. (2009). Relationships between individualismcollectivism, gender, and direct or indirect aggression: A study in China, Poland and the US. Aggressive Behavior, 35(1), 24-30.

Fry, D.P. (2001). Anthropological perspectives on aggression: Sex differences and cultural variation. Aggressive Behavior, 24(2), 81-95.

Fujihara, T., Kohyama, T., Andreu, J.M. y Ramírez, J.M. (1999). Justification of interpersonal aggression in Japanese, American and Spanish students. Aggressive Behavior, 25(3), 185-195.

Gouveia, V.V. (1998). España desde fuera: el individualismo-colectivismo como dimensión cultural. Sociedad y Utopía, 11, 151-166. 
Hambleton, R.K. y Kanjee, A. (1995). Increasing the validity of cross-cultural assessments: Use of improved methods for test adaptions. European Journal of Psychological Assessment, 11(3), 147-157.

Hofstede, G. (1984). The cultural relativity of the quality of life concept. Academy of Management Review, 9(3), 389-398.

Inderbitzen, H.M. y Foster, S.L. (1992). The Teenage Inventory of Social Skills: Development, reliability, and validity. Psychological Assessment, 4(4), 451-459.

Inglés, C.J., Hidalgo, M.D., Méndez, F.X. y Inderbitzen, H.M. (2003). The Teenage Inventory of Social Skills: Reliability and validity of the Spanish translation. Journal of Adolescence, 26(4), 505-510.

Inglés, C.J., Martínez-Monteagudo, M.C., Delgado, B., Torregrosa, M.S., Redondo, J., Benavides, G., García-Fernández, J.M. y García-López, L.J. (2008). Prevalencia de la conducta agresiva, conducta prosocial y ansiedad social en una muestra de adolescentes españoles: un estudio comparativo. Infancia y Aprendizaje, 31(4), 449-461.

Österman, K., Björkqvist, K., Lagerspetz, K.M.J., Kaukiainen, A., Landau, S., Fraczek, A. y Caprara, G.V. (1998). Cross-cultural evidence of female indirect aggression. Aggressive Behavior, 24(1), 1-8.

Oyserman, D., Coon, H.M. y Kemmelmeier, M. (2002). Rethinking individualism and collectivism: Evaluation of theoretical assumptions and meta-analyses. Psychological Bulletin, 128 (1), 3-71.

Páez, D., Fernández, I., Basabe, N. y Grad, H. (2001). Valores culturales y motivacionales: creencias de auto-concepto de Singelis, actitudes de competición de Triandis, control emocional e individualismo-colectivismo vertical-horizontal. Revista Española de Motivación y Emoción, 4(8-9), 169-195.

Pastorelli, C., Barbaranelli, C., Cermark, I., Rozsa, S. y Caprara, G.V. (1997). Measuring emotional instability, prosocial behavior, and aggression in pre-adolescents: A cross national study. Personality and Individual Differences, 23(4), 691-703.

Ramírez, J.M. (1993). Acceptability of aggression in four Spanish regions and a comparison with other European countries. Aggressive Behavior, 19(3), 185-197.

Ramírez, J.M., Andreu, J.M. y Fujihara, T. (2001). Cultural and sex differences in aggression: A comparison between Japanese and Spanish students using two different inventories. Aggressive Behavior, 27(4), 313-322.

Shavelson, J., Hubner, J.J. y Stanton, G.C. (1976). Self-concept: Validation of construct interpretations. Review of Educational Research, 46(3), 407-442.

Toldos, M.P. (2005). Sex and age differences in self-estimated physical, verbal and indirect aggression in Spanish adolescents. Aggressive Behavior, 31(1), 13-23.

Recibido: 10 de enero de 2010

Recepción Modificaciones: 19 de enero de 2010

Aceptado: 22 de enero de 2010 